Exploring unusual bodily experiences, basic self disturbances and multimodal hallucinations in the non-clinical population: a cross-sectional study

Lucretia Thomas $^{1 *}$, Renate Reniers ${ }^{2}$, Lénie Torregrossa ${ }^{3}$ and Clara Humpston ${ }^{4}$

${ }^{1}$ Medical School, College of Medical and Dental Sciences, University of Birmingham; ${ }^{2}$ Institute of Clinical Sciences, University of Birmingham, Institute for Mental Health, School of Psychology, University of Birmingham, Centre for Human Brain Health, University of Birmingham; ${ }^{3}$ Department of Psychology, Vanderbilt University and ${ }^{4}$ Institute for Mental Health, School of Psychology, University of Birmingham

${ }^{*}$ Corresponding author.

doi: 10.1192/bjo.2021.784

Aims. Psychosis research has largely focused on symptoms which are easier to define. Symptoms which are challenging to detect and articulate, including disturbances in the basic- and bodilyself, may not be volunteered by patients, despite causing significant distress. Increased understanding of such symptoms, which may present in the prodromal phase of psychosis and persist following the remission of positive symptoms, may allow patients who experience these to be better supported.

This study aims to explore how disturbances in the basic- and bodily-self relate to multimodal hallucinations. Through sampling a non-clinical population, this study takes the continuum approach to psychosis, where individuals experience sub-clinical psychotic symptoms which do not cause distress or functional impairment.

It is hypothesised that individuals with greater hallucination proneness will exhibit greater severity of ambiguous and imprecise mapping of bodily experiences, and will report greater levels of basic and bodily-self disturbance. This project also aims to evaluate Audiograph as a newly developed tool for creating representations of visual hallucinations.

Method. This is a two-stage cross-sectional study. In stage one, participants completed the Multi-Modality Unusual Sensory Experiences Questionnaire to assess hallucination-proneness. In stage two, all participants were invited to complete seven further validated questionnaires which assessed basic- and bodily selfdisturbances alongside co-variates including anxiety and depression symptoms, delusion-proneness and loneliness. Participants also completed emBODY, a computer-based task which allows participants to map the bodily sensations they experience during 13 different emotional states. Participants with high-hallucination proneness also completed the Audiograph task. Hierarchical linear regression, conducted using Stata, will be used to model the influence of hallucination proneness on measures of basic- and bodily-self disturbance. MATLAB will be used to generate topographical maps of the data from emBODY; maps will be compared between different emotional states using linear discriminant analysis, and between high and low hallucination proneness groups using Spearman's test.

Result. Currently, 50 of the 104 stage one participants have completed stage two.

Since this project comprises a compulsory component of the presenting author's intercalated degree, data collection will cease on the 29th of March in advance of their poster and write-up submission deadlines in May.

Conclusion. Although basic- and bodily-self disturbances have been assessed in previous studies using various techniques, no single study has assessed these alongside multimodal hallucinations to link these concepts together as a whole, especially not in a general population sample. The added value of this project is to precisely address this gap in knowledge.

\section{A systematic review of the effects of nicotine replacement therapy on agitation among nicotine-dependent psychiatric inpatients}

Joseph Toms ${ }^{1 \star}$ and Jacob King ${ }^{2}$

${ }^{1}$ Queen Elizabeth Hospital King's Lynn NHS Foundation Trust and ${ }^{2}$ University College London Hospitals NHS Foundation Trust ${ }^{\star}$ Corresponding author.

doi: 10.1192/bjo.2021.785

Aims. This systematic review aims to evaluate the effect of nicotine replacement therapies (NRTs) on measures of agitation amongst nicotine-dependent adult psychiatric inpatients.

Background. Since the introduction of the smoke-free policy for all psychiatric facilities, a psychiatric admission is likely to upset a nicotine-dependent individual's normal routine of nicotine consumption. In addition to the physiological effects of nicotine withdrawal $(\mathrm{NW})$, the interpersonal dynamic which nurse-led guardianship of nicotine products constructs presents stressors to the nicotine dependent patient.

Several systematic reviews evaluating changes in objective measures of agitation amongst smoking patients in medical critical care units have found varied results, with some demonstrating worsening agitation with NRT use. We therefore believe that there is sufficient equipoise in the use of NRT to prompt a review of studies amongst psychiatric inpatients.

Method. This review identified English language studies through developed search strategies in PubMed/MEDLINE, EMBASE, PyschINFO, PSYCHLit, Cochrane databases, and Google scholar. The bibliographies of notable papers were explored. Hand searches of five major psychiatric journals were conducted. Peer reviewed studies of any study design were included if they reported primary data of adult psychiatric inpatients. Studies were extracted from 1990 - present, this was felt appropriate as nicotine replacement patches became available in 1992.

Search strategies were informed by $\mathrm{MeSH}$ search terms and included multiple conceptions of "agitation", including variations on; agitation, irritability, and arousal to capture the concept from broad academic constructions. The quality of studies was assessed with the Newcastle-Ottawa and Cochrane Collaboration tools.

This review follows PRISMA guidelines, and an application for PROSPERO registration has been submitted pending acceptance. Result. Two studies were identified which matched inclusion criteria. A double-blinded randomised placebo-controlled trial of 40 nicotine-dependent inpatients from Allen et al. reported a significant 23\% reduction in Agitated Behaviour Scores at 24 hours following NRT administration on admission compared to their matched placebo controls. Yet a retrospective cross-sectional analysis from Okoli using scores for NW identified more severe withdrawal symptoms including "restlessness" and "anger/irritability" than nicotine-dependent patients not provided with NRT.

Conclusion. Despite considerable commentary within literature there is presently only one study providing moderate evidence of a positive benefit to measures of agitated behaviour from the use of NRT amongst nicotine-dependent psychiatric inpatients. There is currently very low evidence whether NRT improves or exacerbates the agitation associated with NW amongst nicotinedependent psychiatric inpatients. 
Telemedicine in addictions feasibility RCT - staff and patient qualitative satisfaction

Dominic Treloar ${ }^{1 \star}$ and Soraya Mayet ${ }^{2}$

${ }^{1}$ Hull York Medical School and ${ }^{2}$ Humber Teaching FT

${ }^{*}$ Corresponding author.

doi: 10.1192/bjo.2021.786

Aims. Opioid dependence has high risks. Opioid substitution treatment (OST) improves outcomes. Addiction specialist prescribers prescribe OST and monitor safety, but nonattendance may lead to worse outcomes. Telemedicine can reduce travel and improve attendance at appointments. Before COVID-19, we started a telemedicine in addictions trial to see if this helped in addictions. We present the qualitative patient and staff experience results.

Method. Health Research Authority approval for randomized controlled feasibility trial of Telemedicine versus Face-to-Face (control) consultations at community addictions semirural service $(2500 \mathrm{~km} 2)$ using a modified Hub-and-Spoke (outreach) model. Adult opioid dependent patients prescribed OST and attending outreach were recruited. Participants received two appointments in randomized group. Telemedicine was delivered using Skype-for-business videoconferencing. Patients attended outreach, saw keyworker for drug testing first, and telemedicine conducted via keyworker's laptop. Addiction prescribers located remotely at Hub. Post-trial research interview conducted assessing patient and staff experience of Telemedicine versus Face-to-Face consultations. Data transcribed, inputted to RedCap Cloud and free-text analysed using qualitative thematic analysis.

Result. Of fifty-nine patient participants, 58 completed a research interview. Patient participants reported similar levels of satisfaction between the Telemedicine and Face to Face groups. The themes generated in relation to Face-to-Face were no difference, easy, kind staff and liking being part of research. For Telemedicine, themes were less travel, good experience, easier to access, good communication, saves time and saves money. For instance, one patient stated 'Clear, easy to access less travel' and another patient stated 'I struggle with travel. I found it easier'. Of 19 staff participant research interviews completed, Staff reported Good or Very Good experience with telemedicine which was equivalent for Face-to-Face consultations. Eleven staff had experience of telemedicine consultations during the trial. They reported similar themes to patients with telemedicine leading to less travel, beneficial to patient care, improves attendance and was innovative technology. One staff member reported satisfaction with telemedicine due to 'Time, travel and money reduction'. When questioned on the downsides to telemedicine, technological issues were mainly related to connection issues and sound issues.

Conclusion. In the first known RCT of Telemedicine versus Face-to-Face consultations for patients with opioid dependence attending prescriber review, we found that both patients and staff were satisfied with telemedicine as compared to face-to-face consultations. Overall themes were reduced travel, saving time and more convenience. This will be very important given the impact of COVID-19 on access to addictions services.

Financial Sponsorship

East Riding CCG

Academic Health Science Network

Resolving the discrepancies of suicide risk in obsessivecompulsive patients: a review of incidence rates and risk factors of suicide and suicide attempts in OCD

Himanshu Tyagi ${ }^{1 \star}$ and Gabriel Bundies ${ }^{2}$

${ }^{1}$ University College London Hospitals NHS Trust, National Hospital for Neurology and Neurosurgery, University College London Institute of Neursology and ${ }^{2}$ University College London

${ }^{*}$ Corresponding author.

doi: 10.1192/bjo.2021.787

Aims. Obsessive-Compulsive Disorder (OCD) describes a mental health condition in which affected patients experience persistent obsessions, which may often, but not always result in compulsions, causing major distress and anxiety. Obsessions are defined as intrusive thoughts with a high emotional valence, whereas compulsion are repetitive actions, which demonstrate the attempt to eliminate obsessive thoughts.

When speaking of OCD, risk of suicide is rarely a topic of concern. There is still no consensus about whether OCD and suicide are associated. Early schools of clinical sciences propose a low risk of harm, which was taught to most mental health practitioners currently working in health services. Moreover, the World Health Organisation currently classifies OCD as the 11th leading cause of nonfatal burden, indicating that despite the potential for causing significant disabilities, OCD does not pose any serious health risks. Contemporary evidence, however, suggests that the risk for suicide may be underestimated.

This literature review aims to cumulate evidence for the risk of suicide in OCD and its associated underlying factors to clarify and resolve the discrepancies that currently exist regarding this topic. Method. To identify eligible studies, the databases MEDLINE(R), PubMed, and PsycINFO are used. Selected studies provide data on suicide rates, attempts, and risk factors. Grey literature is included in the review to consider results from studies which may not have qualified for publishing. This literature review is conducted according to the PRISMA guidelines.

Result. After deduplication, 653 studies could be found out of which 15 studies meet the inclusion criteria. Rates of suicide attempts appear to lie between $12 \%$ and $27 \%$. Death-to-suicide rates in OCD are shown to range from $0.7 \%$ to $1.4 \%$. Associated risk factors for suicide in OCD include, mistrust and unacceptable thoughts, depression, and comorbid substance use disorders. The strongest predictor for death caused by suicide is having a history of previous suicide attempts. Higher education and comorbid anxiety disorders act as protective factors. Lastly, gender differences remain unclear since some studies classify female sex as a protective, and some as a risk factor.

Conclusion. This review provides a good overview of the actual risk for suicide in OCD. Current evidence suggests high suicidality in patients with OCD, leading to suicide attempts in affected patients, but not necessarily resulting in death, as the death-to-suicide rates are low. Genetic heritage and comorbidities of further mental health disorders may increase the risk for suicide in OCD.

Pharmacological management of tourette's syndrome comorbid with obsessive-compulsive disorder in adult patients

Himanshu Tyagi* and Olabisi Ogunbiyi

National Hospital for Neurology and Neurosurgery

${ }^{\star}$ Corresponding author.

doi: $10.1192 /$ bjo.2021.788

Aims. Tourette's Syndrome (TS) is a neurodevelopmental disorder, which often presents in childhood and is hallmarked by motor and vocal tics. Obsessive-Compulsive Disorder (OCD) is a chronic neuropsychiatric condition characterised by intrusive thoughts and time-consuming repetitive behaviours. Research suggests that $15-20 \%$ of adult patients with TS will also meet 